\title{
Oxidative dearomatic approach towards the synthesis of erythrina skeleton: a formal synthesis of demethoxyerythratidinone
}

\author{
Zhi-Qiang Pan, Ji-Xuan Liang, Jing-Bo Chen, Xiao-Dong Yang, * and Hong-Bin Zhang* \\ Key Laboratory of Medicinal Chemistry for Natural Resource (Yunnan University), Ministry of Education, School of Chemical \\ Science and Technology, Yunnan University, Kunming 650091, Yunnan, China
}

Received 9 December 2011; Accepted 19 December 2011

(C) The Author(s) 2011. This article is published with open access at Springerlink.com

\begin{abstract}
A concise synthetic route leading to highly functional erythrina alkaloid skeletons has been developed. The key process is an oxidative carbon-carbon coupling followed by a conjugated addition. Based on this new strategy, a formal synthesis of demethoxyerythratidinone was completed in only 6 steps from 4-aminophenol.
\end{abstract}

Keywords: erythrina alkaloid, oxidative dearomation, demethoxyerythratidinone, formal synthesis

\section{Introduction}

The genus Erythrina is widely distributed in tropical and subtropical regions and has been used in indigenous medicine. ${ }^{1}$ Erythrina alkaloids are common constitutes isolated from this species. Representative structures of the erythrina alkaloid family are characterized by a tetracyclic ring system and featured with an 1-azaspiro[5.5] undecane unit (Figure 1). A variety of biological effects are associated with the erythrina skeleton including sedative, hypotensive, neuromuscular blocking and CNS activity. ${ }^{1 \mathrm{a}, 2}$ Because of its typical structure and interesting bioactivities, there has been much interest in the synthesis of these alkaloids and derivatives over the years. ${ }^{3}$ An impressive strategy towards erythrina skeleton is biomimetic approach through an oxidative carbon-carbon bond formation. ${ }^{4}$ Although numerous imaginative synthetic routes have been developed based on the oxidative coupling, ${ }^{5}$ few meet adequate measures of flexibility and efficiency in terms of diversity oriented synthesis.

We recently initiated a research program towards the synthesis of structurally diverse compounds by using oxidative formation of carbon-carbon bond as the key reaction. The spiro cyclohexyldienonyl $\beta$-lactams, oxindoles and structural unit for erythrina alkaloids were synthesized from the amide derivatives of 4-aminophenol. ${ }^{6}$ In this paper, we reported the results towards the synthesis of analogues of erythrina alkaloids.

\section{Results and Discussion}

The retrosynthetic analysis is showed in Scheme 1. Starting from the commercially available 4-aminophenol ( $1 \mathrm{~kg} / 157 \mathrm{USD})$,

*To whom correspondence should be addressed. E-mail: zhanghb@ynu.edu.cn; xdyang@ynu.edu.cn

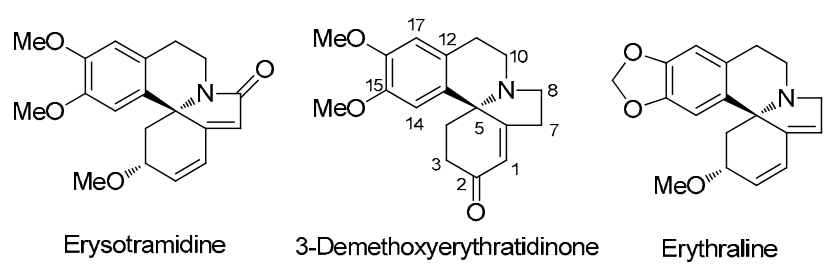

Figure 1. Representative erythrina alkaloids.

we speculated that a highly functional erythrina skeleton could be reached by a sequential oxidative dearomatization and a Michael addition.

We began our research by an amidation of 4-aminophenol (1) with homoveratroyl chloride (2). ${ }^{7}$ The product, amide (3), was then converted to amine by a reduction with lithium aluminium hydride in THF (83\%). By treatment of amine (4) with ethyl 3-chloro-3-oxopropanoate in dichloromethane in the presence of pyridine, amide (5) was obtained in $71 \%$ yield over three steps. Other amides (10 and 11), aiming at the synthesis of erythraline and its analogues, were prepared in three or four steps by the same procedure as shown in Scheme 2.

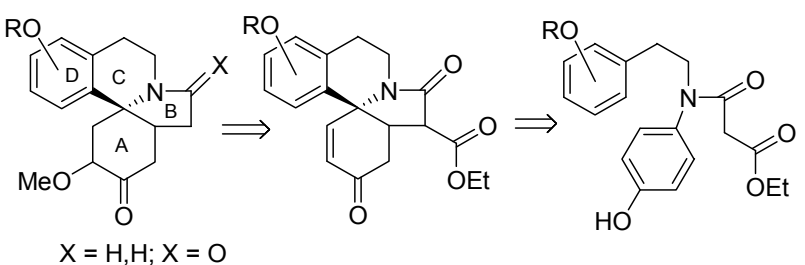

Scheme 1. Retrosynthetic analysis of the erythrina alkaloids. 

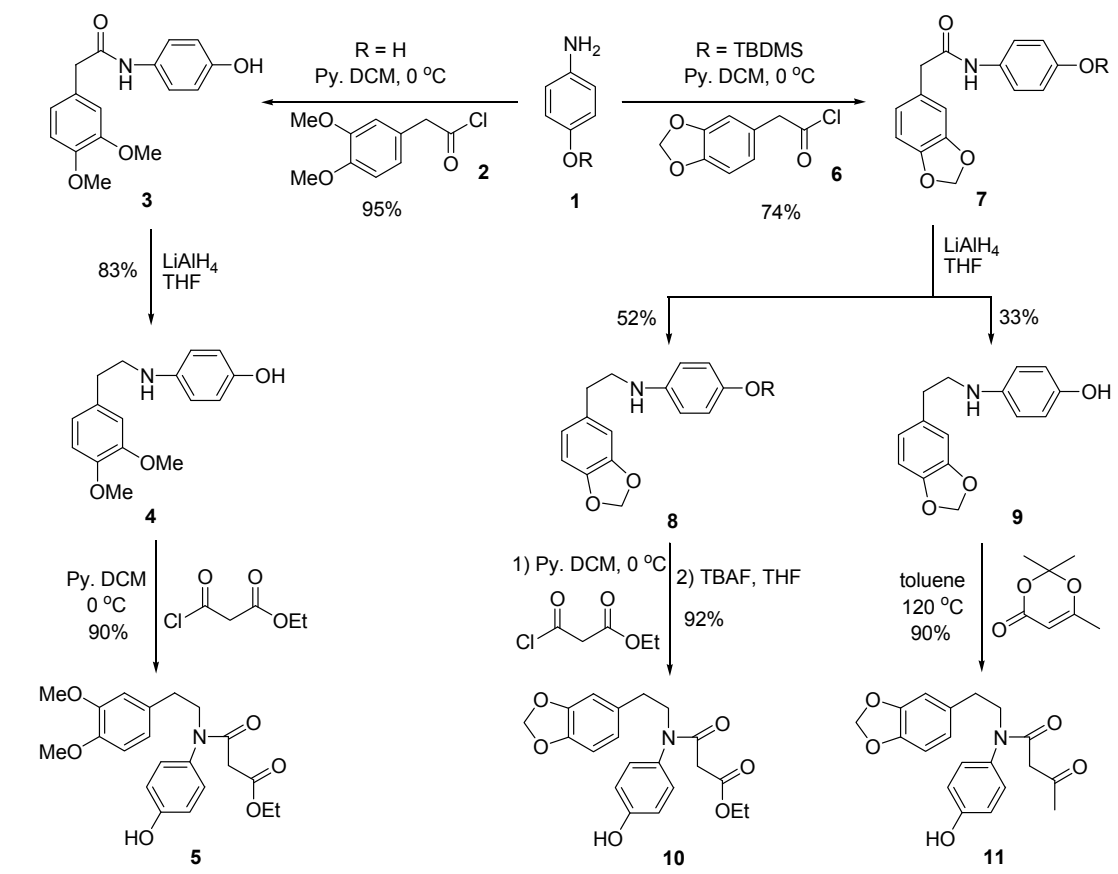

Scheme 2. Synthesis of amide intermediates.

The key oxidative coupling process was attempted by treatment of amide (5) with iodobenzene diacetate (IBD) in methanol, a well-established dearomatization procedure. ${ }^{8}$ To our disappointment, this oxidative condition only led to cyclohexyldienone (12). We then tried the oxidation with (bis(trifluoroacetoxy)iodo) benzene (PIFA) in trifluoroethanol at $-40^{\circ} \mathrm{C}{ }^{9}$ To our delight, this oxidation provided the desire azaspiro cyclohexyldienone (13), after treatment with DBU in dichloromethane, the desire intermediate (14) with a highly functional erythrina skeleton was obtained in $72 \%$ yield. This two steps procedure was soon optimized to be an efficient "one-pot" reaction: after the oxidative coupling, potassium carbonate was directly introduced to the reaction mixture at room temperature, and compound $\mathbf{1 4}$ was obtained in $83 \%$ isolated yield. Although oxidative coupling of amides $\mathbf{1 0}$ and 11 proceeded under the same reaction condition, the yield (70\% and $72 \%$ for $\mathbf{1 5}$ and $\mathbf{1 6}$ ) was relatively lower than that of amide $\mathbf{5}$, possibly due to the poor electron donating ability imposed by stereoelectronic effect of the methylenedioxy substituent. ${ }^{10}$ This one-pot cyclization furnished the highly functional erythrina skeletons in an efficient way (4 steps for erythrina derivative 14 and $4 \sim 5$ steps for erythrina derivatives 15 and 16).

Having successfully constructed the azaspiro cyclohexyldienone, we turned our attention next to convert compound $\mathbf{1 4}$ to the natural product, namely 3-demethoxyerythratidinone. Compound 14 was further manipulated, hydrogenation followed by decarboxylation, to a known intermediate for the synthesis of demethoxyerythratidinone (Scheme 4), thus furnished a formal synthesis of this natural erythrina alkaloid. ${ }^{11}$

In conclusion, we have developed a practical and efficient method for the synthesis of erythrina skeletons. Based on this methodology, a formal synthesis of demethoxyerythratidinone was also completed. The highly functional erythrina derivatives could be used not only as intermediates for the synthesis of natural erythrina alkaloids but also be used as building blocks for the synthesis of erythrina alkaloid-like compounds.
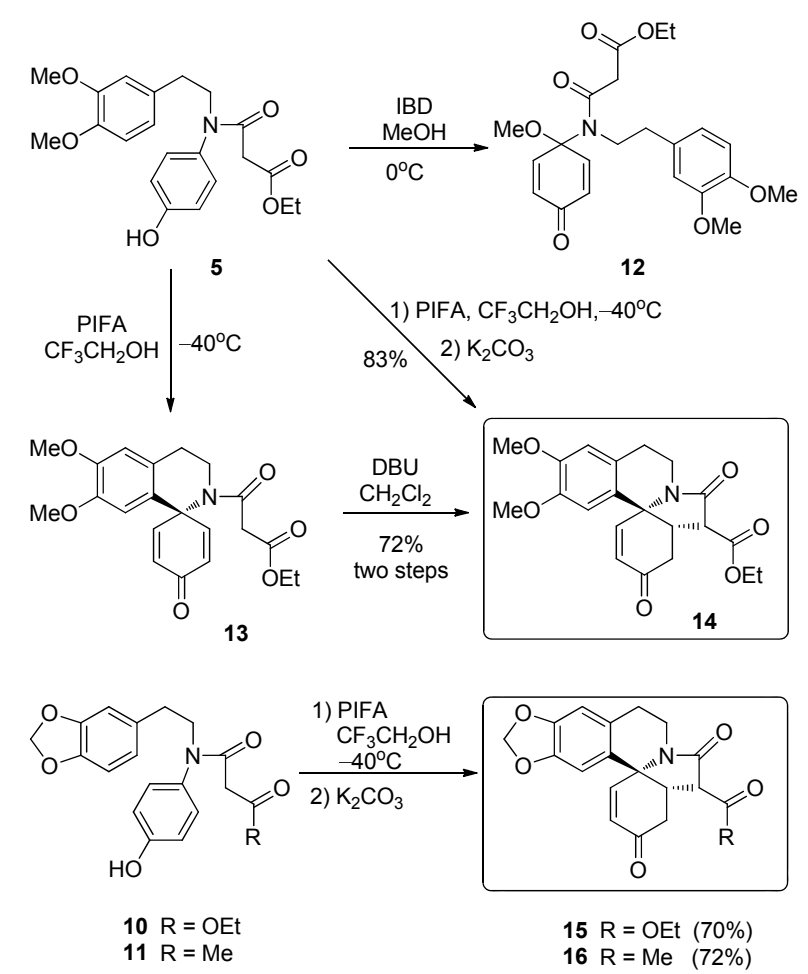

Scheme 3. Concise synthesis of erythrina skeletons.

\section{照 Springer}


<smiles>CCOC(=O)CC1CC(=O)C=CC12c1cc(OC)c(OC)cc1CCN2C(=O)O</smiles>

14

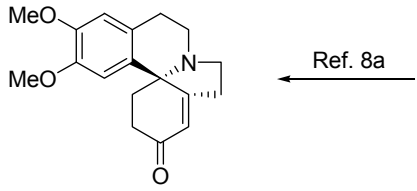

Demethoxyerythratidinone

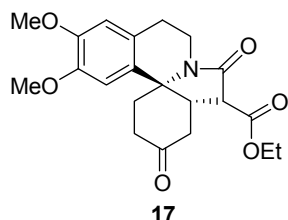

17
Scheme 4. Formal synthesis of erythrina alkaloid demethoxyerythratidinone.

\section{Experimental Section}

General Experimental Procedures. Melting points were obtained on a XT-4 melting-point apparatus and were uncorrected. Proton nuclear magnetic resonance ( ${ }^{1} \mathrm{H}$ NMR) spectra were recorded on a Bruker Avance 300 spectrometer at $300 \mathrm{MHz}$. Carbon-13 nuclear magnetic resonance $\left({ }^{13} \mathrm{C} \mathrm{NMR}\right)$ was recorded on Bruker Avance 300 spectrometer at $75 \mathrm{MHz}$. Chemical shifts are reported as $\delta$ values in parts per million (ppm) relative to tetramethylsilane (TMS) for all recorded NMR spectra. Low-resolution mass spectra were recorded on a VG Auto Spec-3000 magnetic sector MS spectrometer. High resolution mass spectra were taken on AB QSTAR Pulsar mass spectrometer. Silica gel (200-300 mesh) for column chromatography and silica $\mathrm{GF}_{254}$ for TLC were produced by Qingdao Marine Chemical Company (China). All air- or moisture-sensitive reactions were conducted under an argon atmosphere. Starting materials and reagents used in reactions were obtained commercially from Acros, Aldrich, Fluka and were used without purification, unless otherwise indicated.

Synthesis of Compound 3. To a solution of 4-aminophenol $(\mathbf{1}, 546 \mathrm{mg}, 5 \mathrm{mmol})$ in $\mathrm{CH}_{2} \mathrm{Cl}_{2}(20 \mathrm{~mL})$ and pyridine $(2.02$ $\mathrm{mL}, 25 \mathrm{mmol}, 5.0$ eq.) at $0^{\circ} \mathrm{C}$, homoveratroyl chloride $(2,5.5$ mmol, 1.1 eq.) in dichloromethane $(5 \mathrm{~mL})$ was added dropwise. The resulting mixture was then stirred at room temperature for 3-4 h. The reaction mixture was diluted with $\mathrm{CH}_{2} \mathrm{Cl}_{2}(20 \mathrm{~mL})$ and treated with $2 \mathrm{~N} \mathrm{HCl}(10 \mathrm{~mL})$ for $10 \mathrm{~min}$ and the aqueous phase was separated and the organic phase was washed with water $(2 \times 15 \mathrm{~mL})$. After dried over anhydrous $\mathrm{Na}_{2} \mathrm{SO}_{4}$ and filtration, the solvent was removed under reduced pressure and the residue $(1.43 \mathrm{~g})$ was used in next step without further purification.

Synthesis of Compound 4. To a solution of $3(1.43 \mathrm{~g})$ in anhydrous THF $(50 \mathrm{~mL})$ at $0^{\circ} \mathrm{C}, \mathrm{LiAlH}_{4}(380 \mathrm{mg}, 10 \mathrm{mmol}$, 2.0 eq.) was added in one portion. The resulting mixture was gradually warmed up to room temperature then stirred at $75^{\circ} \mathrm{C}$ overnight. The reaction mixture was cooled to $0^{\circ} \mathrm{C}$ and an aqueous solution of saturated $\mathrm{K}_{2} \mathrm{CO}_{3}(5 \mathrm{~mL})$ was added dropwise. The mixture was diluted with water $(50 \mathrm{~mL})$ and extracted with EtOAc $(3 \times 15 \mathrm{~mL})$. The organic phases were combined and washed with water $(2 \times 15 \mathrm{~mL})$ and brine $(15$ $\mathrm{mL}$ ). After dried over anhydrous $\mathrm{Na}_{2} \mathrm{SO}_{4}$ and filtration, the solvent was removed under reduced pressure and the residue was chromatographed on silica gel $\left(\mathrm{CH}_{2} \mathrm{Cl}_{2}:\right.$ EtOAc $\left.=15: 1\right)$ to afford amine 4 (984 mg, 72\% over two steps). Pale yellow syrup, ${ }^{1} \mathrm{H}$ NMR $\left(300 \mathrm{MHz}, \mathrm{CD}_{3} \mathrm{COCD}_{3}\right) \delta: 6.82(1 \mathrm{H}, \mathrm{d}, J=$ $8.1 \mathrm{~Hz}), 6.80-6.63(4 \mathrm{H}, \mathrm{m}), 6.54(2 \mathrm{H}, \mathrm{d}, J=8.4 \mathrm{~Hz}), 3.85(3 \mathrm{H}$, s), $3.84(3 \mathrm{H}, \mathrm{s}), 3.32(1 \mathrm{H}, \mathrm{t}, J=6.6 \mathrm{~Hz}), 2.83(1 \mathrm{H}, \mathrm{t}, J=6.6$ $\mathrm{Hz}) .{ }^{13} \mathrm{C} \mathrm{NMR}\left(75 \mathrm{MHz}, \mathrm{CD}_{3} \mathrm{COCD}_{3}\right) \delta: 148.98,148.71$, $147.60,141.46,131.86,120.78,116.36,115.43,112.16$, $111.52,55.96,55.87,46.61,34.93$. HRESIMS $\mathrm{m} / \mathrm{z}$ found: 274.1437, calcd. for $\mathrm{C}_{16} \mathrm{H}_{20} \mathrm{NO}_{3}[\mathrm{M}+\mathrm{H}]^{+}: 274.1443$.

Synthesis of Compound 5. To a solution of 4 (820 mg, 3 $\mathrm{mmol})$ in $\mathrm{CH}_{2} \mathrm{Cl}_{2}(30 \mathrm{~mL})$ and pyridine $(1.21 \mathrm{~mL}, 15 \mathrm{mmol}$, 5.0 eq.) at $0^{\circ} \mathrm{C}$, ethyl 3-chloro-3-oxopropanoate $(496 \mathrm{mg}, 3.3$ mmol, 1.1 eq.) in $\mathrm{CH}_{2} \mathrm{Cl}_{2}(3 \mathrm{~mL})$ was added dropwise. The resulting mixture was then stirred at room temperature for 2-3 h. The reaction mixture was then treated with $2 \mathrm{~N} \mathrm{HCl}(10 \mathrm{~mL})$ for $10 \mathrm{~min}$ and diluted with $\mathrm{CH}_{2} \mathrm{Cl}_{2}(20 \mathrm{~mL})$. The aqueous phase was separated and the organic phase was washed with water $(2 \times 3 \mathrm{~mL})$. The organic phases were combined and back extracted with EtOAc $(10 \mathrm{~mL})$. The combined organic phases were dried over anhydrous $\mathrm{Na}_{2} \mathrm{SO}_{4}$ and filtration. After removal of the solvent under reduced pressure, the residue was chromatographed on silica gel (Hexane : EtOAc $=4: 1-2: 1)$ to afford amides $5(815 \mathrm{mg}, 70 \%)$. Pale yellow powder, m.p.: $138-139^{\circ} \mathrm{C} .{ }^{1} \mathrm{H}$ NMR $\left(300 \mathrm{MHz}, \mathrm{CDCl}_{3}\right) \delta: 6.93(2 \mathrm{H}, \mathrm{d}, J=$ $8.7 \mathrm{~Hz}), 6.90(2 \mathrm{H}, \mathrm{d}, J=8.7 \mathrm{~Hz}), 6.75(1 \mathrm{H}, \mathrm{d}, J=7.8 \mathrm{~Hz})$, $6.74(1 \mathrm{H}, \mathrm{d}, J=1.5 \mathrm{~Hz}), 6.68(1 \mathrm{H}, \mathrm{dd}, J=7.8,1.5 \mathrm{~Hz}), 4.10$ $(2 \mathrm{H}, \mathrm{q}, J=7.2 \mathrm{~Hz}), 3.89(2 \mathrm{H}, \mathrm{t}, J=7.9 \mathrm{~Hz}), 3.81(6 \mathrm{H}, \mathrm{s}), 3.20$ $(2 \mathrm{H}, \mathrm{s}), 2.83(2 \mathrm{H}, \mathrm{t}, J=7.9 \mathrm{~Hz}), 1.20(3 \mathrm{H}, \mathrm{t}, J=7.2 \mathrm{~Hz}) \cdot{ }^{13} \mathrm{C}$ NMR $\left(75 \mathrm{MHz}, \mathrm{CDCl}_{3}\right) \delta$ : 167.97, 167.14, 157.11, 148.97, $147.64,133.75,131.09,129.17,120.87,116.73,112.22$, $111.42,61.51,55.98,55.92,51.50,41.90,33.37,14.09$. HRESIMS $\mathrm{m} / \mathrm{z}$ found: 388.1759 , calcd. for $\mathrm{C}_{21} \mathrm{H}_{26} \mathrm{NO}_{6}[\mathrm{M}+$ $\mathrm{H}^{+}: 388.1760$.

Synthesis of Compounds 8 and 9. The procedures were same as compounds 3 and 4 . Compound $\mathbf{8}(52 \%)$ : Pale yellow syrup. ${ }^{1} \mathrm{H}$ NMR $\left(300 \mathrm{MHz}, \mathrm{CD}_{3} \mathrm{COCD}_{3}\right) \delta: 6.59(1 \mathrm{H}, \mathrm{d}, J=$ $8.1 \mathrm{~Hz}), 6.55-6.51(4 \mathrm{H}, \mathrm{m}), 6.34(2 \mathrm{H}, \mathrm{d}, J=8.4 \mathrm{~Hz}), 5.76(2 \mathrm{H}$, s), $3.12(1 \mathrm{H}, \mathrm{t}, J=6.9 \mathrm{~Hz}), 2.64(1 \mathrm{H}, \mathrm{t}, J=6.9 \mathrm{~Hz}), 0.83(9 \mathrm{H}$, s), $0.00(9 \mathrm{H}, \mathrm{s}) .{ }^{13} \mathrm{C} \mathrm{NMR}\left(75 \mathrm{MHz}, \mathrm{CD}_{3} \mathrm{COCD}_{3}\right) \delta: 147.82$, $1447.54,146.12,142.54,133.19,121.66,120.71,114.20$, $109.12,108.32,100.87,46.11,35.37,25.79,18.20,-4.50$. HRESIMS $m / z$ found: 371.1925 , calcd. for $\mathrm{C}_{21} \mathrm{H}_{29} \mathrm{NO}_{3} \mathrm{Si}[\mathrm{M}]^{+}$: 371.1917. Compound 9 (33\%): Pale yellow syrup, ${ }^{1} \mathrm{H}$ NMR $\left(300 \mathrm{MHz}, \mathrm{CD}_{3} \mathrm{COCD}_{3}\right) \delta: 6.75(1 \mathrm{H}, \mathrm{d}, J=7.8 \mathrm{~Hz}), 6.70-6.63$ $(4 \mathrm{H}, \mathrm{m}), 6.53(2 \mathrm{H}, \mathrm{d}, J=8.7 \mathrm{~Hz}), 5.93(2 \mathrm{H}, \mathrm{s}), 3.29(1 \mathrm{H}, \mathrm{t}, J=$ $6.9 \mathrm{~Hz}), 2.80(1 \mathrm{H}, \mathrm{t}, J=6.9 \mathrm{~Hz}) .{ }^{13} \mathrm{C}$ NMR $(75 \mathrm{MHz}$, $\left.\mathrm{CD}_{3} \mathrm{COCD}_{3}\right) \delta: 147.97,148.80,146.11,142.05,133.08$, $121.65,116.25,114.77,109.09,108.32,100.86,46.35,35.25$. HRESIMS $\mathrm{m} / \mathrm{z}$ found: 257.1057 , calcd. for $\mathrm{C}_{15} \mathrm{H}_{15} \mathrm{NO}_{3}[\mathrm{M}]^{+}$: 257.1052 .

Synthesis of Compound 10. To a solution of $8(557 \mathrm{mg}$, $1.5 \mathrm{mmol})$ in $\mathrm{CH}_{2} \mathrm{Cl}_{2}(20 \mathrm{~mL})$ and pyridine $(0.6 \mathrm{~mL}, 7.5 \mathrm{mmol}$, 5.0 eq.) at $0^{\circ} \mathrm{C}$, ethyl 3-chloro-3-oxopropanoate (248 mg, 1.65 mmol, 1.1 eq.) in $\mathrm{CH}_{2} \mathrm{Cl}_{2}(3 \mathrm{~mL})$ was added dropwise. The 
resulting mixture was then stirred at room temperature for 2-3 $\mathrm{h}$. The reaction mixture was then treated with $2 \mathrm{~N} \mathrm{HCl}(10 \mathrm{~mL})$ for $10 \mathrm{~min}$ and diluted with $\mathrm{CH}_{2} \mathrm{Cl}_{2}(20 \mathrm{~mL})$. The aqueous phase was separated and the organic phase was washed with water $(2 \times 3 \mathrm{~mL})$. The organic phases were combined and back extracted with EtOAc $(10 \mathrm{~mL})$. The combined organic phases were dried over anhydrous $\mathrm{Na}_{2} \mathrm{SO}_{4}$ and filtration. Then removal of the solvent under reduced pressure and dissolved in dry THF $(20 \mathrm{~mL})$. To the solution was added TBFA $(1.0 \mathrm{M}$ solution in THF, $1.5 \mathrm{~mL}, 1.5 \mathrm{mmol}$ ) under argon. The reaction mixture was stirred at room temperature for $2 \mathrm{~h}$. The mixture was concentrated and diluted with water $(30 \mathrm{~mL})$ and extracted with EtOAc $(3 \times 15 \mathrm{~mL})$. The organic phases were combined and washed with water $(2 \times 15 \mathrm{~mL})$ and brine $(15 \mathrm{~mL})$. After dried over anhydrous $\mathrm{Na}_{2} \mathrm{SO}_{4}$ and filtration, the solvent was removed under reduced pressure and the residue was chromatographed on silica gel (Hexane : EtOAc $=4: 1-2: 1)$ to afford amine 10 (512 $\mathrm{mg}, 92 \%$ over two steps). Pale yellow powder. ${ }^{1} \mathrm{H}$ NMR $\left(300 \mathrm{MHz}, \mathrm{CDCl}_{3}\right) \delta: 8.10(1 \mathrm{H}, \mathrm{s}), 7.00(2 \mathrm{H}$, $\mathrm{d}, J=8.7 \mathrm{~Hz}), 6.94(2 \mathrm{H}, \mathrm{d}, J=8.7 \mathrm{~Hz}), 6.68(1 \mathrm{H}, \mathrm{d}, J=7.8$ $\mathrm{Hz}), 6.65(1 \mathrm{H}, \mathrm{d}, J=1.2 \mathrm{~Hz}), 6.60(1 \mathrm{H}, \mathrm{dd}, J=1.2,7.8 \mathrm{~Hz})$, $5.88(1 \mathrm{H}, \mathrm{s}), 4.12(2 \mathrm{H}, \mathrm{q}, J=8.1 \mathrm{~Hz}), 3.86(2 \mathrm{H}, \mathrm{t}, J=8.4 \mathrm{~Hz})$, $3.23(2 \mathrm{H}, \mathrm{s}), 2.81(2 \mathrm{H}, \mathrm{t}, J=8.1 \mathrm{~Hz}), 1.25(3 \mathrm{H}, \mathrm{t}, J=7.2 \mathrm{~Hz})$. ${ }^{13} \mathrm{C}$ NMR $\left(75 \mathrm{MHz}, \mathrm{CDCl}_{3}\right) \delta: 167.91,167.19,157.11,147.64$, $146.09,133.58,132.12,129.01,121.68,116.75,109.22$, 108.22, 100.81, 61.47, 51.66, 41.79, 33.45, 14.01. HRESIMS $\mathrm{m} / \mathrm{z}$ found: 371.1369 , calcd. for $\mathrm{C}_{20} \mathrm{H}_{21} \mathrm{NO}_{6}[\mathrm{M}]^{+}: 371.1369$.

Synthesis of Compound 11. To a solution of 9 (386 mg, $1.5 \mathrm{mmol})$ in toluene $(20 \mathrm{~mL})$ 2,2,6-trimethyl-1,3-dioxin-4-one (213 $\mathrm{mg}, 1.5 \mathrm{mmol})$ in toluene $(5 \mathrm{~mL})$ was added dropwise. The resulting mixture was then stirred at reflux overnight. The mixture was concentrated and the residue was chromatographed on silica gel (Hexane : EtOAc $=4: 1-2: 1)$ to afford amine 11 (423 mg, 90\%). Pale yellow powder. ${ }^{1} \mathrm{H}$ NMR $\left(300 \mathrm{MHz}, \mathrm{CDCl}_{3}\right) \delta: 7.91(1 \mathrm{H}, \mathrm{s}), 6.95(2 \mathrm{H}, \mathrm{d}, J=8.7 \mathrm{~Hz})$, $6.91(2 \mathrm{H}, \mathrm{d}, J=8.7 \mathrm{~Hz}), 6.72(1 \mathrm{H}, \mathrm{d}, J=8.1 \mathrm{~Hz}), 6.66(1 \mathrm{H}, \mathrm{d}$, $J=1.2 \mathrm{~Hz}), 6.61(1 \mathrm{H}, \mathrm{dd}, J=1.2,8.1 \mathrm{~Hz}), 5.91(1 \mathrm{H}, \mathrm{s}), 3.87$ $(2 \mathrm{H}, \mathrm{q}, J=7.8 \mathrm{~Hz}), 3.33(2 \mathrm{H}, \mathrm{s}), 2.81(2 \mathrm{H}, \mathrm{t}, J=7.8 \mathrm{~Hz}), 2.06$ $(3 \mathrm{H}, \mathrm{s}) .{ }^{13} \mathrm{C}$ NMR $\left(75 \mathrm{MHz}, \mathrm{CDCl}_{3}\right) \delta: 202.64,167.77,156.90$, $147.65,146.09,133.82,132.13,129.09,121.70,116.70$, 109.23, 108.23, 100.82, 51.39, 50.13, 33.52, 30.33. HRESIMS $m / z$ found: 341.1259 , calcd. for $\mathrm{C}_{19} \mathrm{H}_{19} \mathrm{NO}_{5}[\mathrm{M}]^{+}: 341.1263$.

Synthesis of Compound 14. To a solution of $5(387 \mathrm{mg}$, $1.0 \mathrm{mmol})$ in $\mathrm{CF}_{3} \mathrm{CH}_{2} \mathrm{OH}(3 \mathrm{~mL})$ was added PIFA $(473 \mathrm{mg}$, $1.1 \mathrm{mmol}, 1.1$ eq.) at $-40^{\circ} \mathrm{C}$ under $\mathrm{N}_{2}$. After stirring at $-40^{\circ} \mathrm{C}$ for $2 \mathrm{~h}$, the reaction mixture was allowed to warm to room temperature then a powder of $\mathrm{K}_{2} \mathrm{CO}_{3}(456 \mathrm{mg}, 3.3 \mathrm{mmol}, 3.3$ eq.) was added. The resulting mixture was filtered and washed with EtOAc $(10 \mathrm{~mL})$. The filtrate was concentrated and the crude products were chromatographed on silica gel $\left(\mathrm{CH}_{2} \mathrm{Cl}_{2}\right.$ : EtOAc $=10: 1)$ to provide enone $14(318 \mathrm{mg}, 83 \%)$. White crystals, m.p.: $161-162^{\circ} \mathrm{C} .{ }^{1} \mathrm{H}$ NMR $\left(300 \mathrm{MHz}, \mathrm{CDCl}_{3}\right) \delta$ : $6.71(1 \mathrm{H}, \mathrm{dd}, J=10.5,1.8 \mathrm{~Hz}), 6.67(1 \mathrm{H}, \mathrm{s}), 6.65(1 \mathrm{H}, \mathrm{s}), 6.18$ $(1 \mathrm{H}, \mathrm{d}, J=10.2 \mathrm{~Hz}), 4.41(1 \mathrm{H}, \mathrm{dd}, J=12.0,3.9 \mathrm{~Hz}), 4.30$ $4.21(2 \mathrm{H}, \mathrm{m}), 3.87(3 \mathrm{H}, \mathrm{s}), 3.79(3 \mathrm{H}, \mathrm{s}), 3.39(1 \mathrm{H}, \mathrm{d}, J=11.7$ $\mathrm{Hz}), 3.21(1 \mathrm{H}, \mathrm{dd}, J=12.1,5.7 \mathrm{~Hz}), 3.20-2.93(3 \mathrm{H}, \mathrm{m}), 2.72$ $(1 \mathrm{H}, \mathrm{d}, J=12.9 \mathrm{~Hz}), 2.66(1 \mathrm{H}, \mathrm{d}, J=12.9 \mathrm{~Hz}), 1.27(3 \mathrm{H}, \mathrm{t}, J$ $=7.2 \mathrm{~Hz}) \cdot{ }^{13} \mathrm{C}$ NMR $\left(75 \mathrm{MHz}, \mathrm{CDCl}_{3}\right) \delta: 195.07,168.29$, $166.65,149.23,148.49,145.79,127.65,126.75,126.30$,
$112.79,108.77,62.19,59.49,56.36,56.09,54.03,44.45$, $36.69,35.73,29.03,14.29$. HRESIMS $\mathrm{m} / z$ found: 386.1599 , calcd. for $\mathrm{C}_{21} \mathrm{H}_{24} \mathrm{NO}_{6}[\mathrm{M}+\mathrm{H}]^{+}: 386.1603$.

Synthesis of Compounds 15 and 16. The procedures were same as compound 14. Compound $15(70 \%)$ : Pale yellow syrup. ${ }^{1} \mathrm{H}$ NMR $\left(300 \mathrm{MHz}, \mathrm{CDCl}_{3}\right) \delta: 6.69(1 \mathrm{H}, \mathrm{dd}, J=10.2$, $1.8 \mathrm{~Hz}), 6.67(1 \mathrm{H}, \mathrm{s}), 6.66(1 \mathrm{H}, \mathrm{s}), 6.17(1 \mathrm{H}, \mathrm{d}, J=10.2 \mathrm{~Hz})$, $5.96(2 \mathrm{H}, \mathrm{s}), 4.40(1 \mathrm{H}, \mathrm{dd}, J=12.0,3.9 \mathrm{~Hz}), 4.31-4.20(2 \mathrm{H}$, $\mathrm{m}), 3.38(1 \mathrm{H}, \mathrm{d}, J=11.7 \mathrm{~Hz}), 3.21(1 \mathrm{H}, \mathrm{m}), 3.13-2.89(3 \mathrm{H}$, $\mathrm{m}), 2.70(1 \mathrm{H}, \mathrm{d}, J=12.9 \mathrm{~Hz}), 2.65(1 \mathrm{H}, \mathrm{d}, J=12.9 \mathrm{~Hz}), 1.31$ $(3 \mathrm{H}, \mathrm{t}, J=7.2 \mathrm{~Hz}) .{ }^{13} \mathrm{C}$ NMR $\left(75 \mathrm{MHz}, \mathrm{CDCl}_{3}\right) \delta: 194.82$, $168.17,166.51,147.64,147.17,145.38,127.81,126.64$, $127.31,109.85,105.57,101.47,62.07,59.63,53.89,44.39$, 36.34, 35.54, 29.46, 14.15. HRESIMS $\mathrm{m} / \mathrm{z}$ found: 369.1220 , calcd. for $\mathrm{C}_{20} \mathrm{H}_{19} \mathrm{NO}_{6}[\mathrm{M}]^{+}$: 369.1220. Compound 16 (72\%): Pale yellow syrup. ${ }^{1} \mathrm{H}$ NMR $\left(300 \mathrm{MHz}, \mathrm{CDCl}_{3}\right) \delta: 6.71(1 \mathrm{H}, \mathrm{s})$, $6.68(1 \mathrm{H}, \mathrm{dd}, J=10.2,1.8 \mathrm{~Hz}), 6.45(1 \mathrm{H}, \mathrm{s}), 6.15(1 \mathrm{H}, d, J=$ $10.2 \mathrm{~Hz}), 5.95(2 \mathrm{H}, \mathrm{s}), 4.34(1 \mathrm{H}, \mathrm{dd}, J=12.0,3.9 \mathrm{~Hz}), 3.48$ $(1 \mathrm{H}, \mathrm{d}, J=11.4 \mathrm{~Hz}), 3.30-3.24(1 \mathrm{H}, \mathrm{m}), 3.13-3.01(2 \mathrm{H}, \mathrm{m})$, $2.88(1 \mathrm{H}, \mathrm{ddd}, J=16.8,12.3,5.1 \mathrm{~Hz}), 2.69(1 \mathrm{H}, \mathrm{dd}, J=15.9$, $2.1 \mathrm{~Hz}), 2.54(1 \mathrm{H}, \mathrm{d}, J=18.3 \mathrm{~Hz}), 2.47(3 \mathrm{H}, \mathrm{s}) .{ }^{13} \mathrm{C} \operatorname{NMR}(75$ $\left.\mathrm{MHz}, \mathrm{CDCl}_{3}\right) \delta: 201.74,195.45,166.55,147.58,147.18$, $145.65,127.68,127.49,109.74,105.55,101.45,59.87,59.13$, $41.48,36.72,35.42,30.97,29.37$. HRESIMS $\mathrm{m} / \mathrm{z}$ found: 339.1104, calcd. for $\mathrm{C}_{19} \mathrm{H}_{17} \mathrm{NO}_{5}[\mathrm{M}]^{+}: 339.1104$.

Synthesis of Compound 17. To a solution of $\mathbf{1 3}(385.4 \mathrm{mg}$, $1.0 \mathrm{mmol})$ in anhydrous EtOH $(20 \mathrm{~mL})$ was added a powder of $10 \% \mathrm{Pd} / \mathrm{C}(38 \mathrm{mg})$. The resulting mixture was then stirred at room temperature under a balloon of hydrogen overnight. After fitration and washed with $\mathrm{CH}_{2} \mathrm{Cl}_{2}(5 \mathrm{~mL})$, the filtrate was concentrated and the residue was chromatographed on silica gel $\left(\mathrm{CH}_{2} \mathrm{Cl}_{2}:\right.$ EtOAc $\left.=5: 1-3: 1\right)$ to afford ketone 17 (368 mg, 95\%). White plates, m.p.: $182-183^{\circ} \mathrm{C} .{ }^{1} \mathrm{H}$ NMR $(300 \mathrm{MHz}$, $\left.\mathrm{CDCl}_{3}\right) \delta: 6.68(1 \mathrm{H}, \mathrm{s}), 6.56(1 \mathrm{H}, \mathrm{s}), 4.34(1 \mathrm{H}, \mathrm{dd}, J=13.2$, $5.4 \mathrm{~Hz}), 4.15(2 \mathrm{H}, \mathrm{q}, J=7.2 \mathrm{~Hz}), 3.86(3 \mathrm{H}, \mathrm{s}), 3.84(3 \mathrm{H}, \mathrm{s})$, $3.44-3.34(1 \mathrm{H}, \mathrm{m}), 3.18(1 \mathrm{H}, \mathrm{d}, J=8.1 \mathrm{~Hz}), 3.15-2.90(2 \mathrm{H}$, $\mathrm{m}), 2.65(1 \mathrm{H}, \mathrm{d}, J=16.8 \mathrm{~Hz}), 2.64(1 \mathrm{H}, \mathrm{d}, J=16.8 \mathrm{~Hz}), 2.49-$ $2.20(4 \mathrm{H}, \mathrm{m}), 1.22(3 \mathrm{H}, \mathrm{t}, J=7.2 \mathrm{~Hz}) .{ }^{13} \mathrm{C}$ NMR $(75 \mathrm{MHz}$, $\left.\mathrm{CDCl}_{3}\right) \delta: 209.23,169.01,167.18,148.71,148.54,133.87$, $125.48,111.94,107.36,62.16,61.47,56.44,56.03,55.21$, $42.55,42.06,35.62,35.44,33.50,27.82,14.14$. HRESIMS $\mathrm{m} / \mathrm{z}$ found: 388.1751 , calcd. for $\mathrm{C}_{21} \mathrm{H}_{26} \mathrm{NO}_{6}[\mathrm{M}+\mathrm{H}]^{+}: 388.1760$.

Synthesis of Compound 18. A mixture of 17 (193 mg, 0.5 mmol.), $\mathrm{LiCl}$ (21 mg, $0.5 \mathrm{mmol}, 1.0$ eq.) and $\mathrm{H}_{2} \mathrm{O}(27 \mathrm{mg}, 1.5$ mmol, 3.0 eq.) in DMSO $(15 \mathrm{~mL})$ was stirred at $158^{\circ} \mathrm{C}$ under $\mathrm{N}_{2}$ overnight (ca. 15-17 h). The resulting mixture was then concentrated under reduced pressure and the residue was chromatographed on silica gel $\left(\mathrm{CH}_{2} \mathrm{Cl}_{2}:\right.$ EtOAc $\left.=3: 1-2: 1\right)$ to afford 18 (118 mg, 75\%). Pale yellow powder, m.p.: 164$165^{\circ} \mathrm{C} .{ }^{1} \mathrm{H}$ NMR $\left(300 \mathrm{MHz}, \mathrm{CDCl}_{3}\right) \delta: 6.68(1 \mathrm{H}, \mathrm{s}), 6.56(1 \mathrm{H}$, s), $4.37(1 \mathrm{H}, \mathrm{dd}, J=12.0,5.4 \mathrm{~Hz}), 3.86(3 \mathrm{H}, \mathrm{s}), 3.84(3 \mathrm{H}, \mathrm{s})$, $3.15-2.90(4 \mathrm{H}, \mathrm{m}), 2.78-2.53(3 \mathrm{H}, \mathrm{m}), 2.49-2.20(4 \mathrm{H}, \mathrm{m})$, $2.11(1 \mathrm{H}, \mathrm{dd}, J=17.8,7.5 \mathrm{~Hz}) .{ }^{13} \mathrm{C}$ NMR $\left(75 \mathrm{MHz}, \mathrm{CDCl}_{3}\right) \delta$ : $210.13,172.19,148.60,148.43,134.48,125.65,111.91$, $107.45,62.56,56.45,56.03,43.38,37.90,37.59,35.35,34.84$, 33.64, 27.67. HRESIMS $\mathrm{m} / \mathrm{z}$ found: 316.1556 , calcd. for $\mathrm{C}_{18} \mathrm{H}_{22} \mathrm{NO}_{4}[\mathrm{M}+\mathrm{H}]^{+}: 316.1548$.

\section{望




\section{Acknowledgments}

This work was supported by grants $(20925205,21062026$, 2010GA014 and 2009CB522300) from National Natural Science Foundation of China, Yunnan Province and National Basic Research Program of China (973 Program).

Open Access This article is distributed under the terms of the Creative Commons Attribution License which permits any use, distribution, and reproduction in any medium, provided the original author(s) and source are credited.

\section{References}

[1] (a) Dyke, S. F.; Quessy, S. N. The Alkaloids; Rodrigo, R. G. A., Ed.; Academic Press: New York, 1981; Vol. 18, pp 1-98. (b) Sano, T.; Tsuda, Y. The Alkaloids; Cordell, G. A., Ed; Academic Press: New York, 1996; Vol. 48, pp 249-337.

[2] Tanaka, H.; Tanaka, T.; Etoh, H.; Goto, S.; Terada, Y. Heterocycles 1999, 51, 2759-2764.

[3] Reimann, E. Synthesis pathways to erythrina alkaloids and erythrina type compounds. in Progress in the chemistry of organic natural products; Herz, W., Falk, H., Kirby, G. W., Ed.; SpringerVerlag/Wien: Austria, 2007; Vol. 88, pp 1-62.

[4] Leete, E.; Ahmad, A. J. Am. Chem. Soc. 1966, 88, 4722-4725.

[5] (a) Quideau, S.; Pouysegu, L.; Deffieux, D. Synlett 2008, 467-495. (b) Ciufolini, M. A.; Braun, N. A.; Canesi, S.; Ousmer, M.; Chang, J.; Chai, D. Synthesis 2007, 3759-3772. (c) Magdziak, D.; Meek, S. J.; Pettus, T. R. R. Chem. Rev. 2004, 104, 1383-1430. (d) Rodrígues, S.; Wipf, P. Synthesis 2004, 2767-2783. (e) Van De Water, R. W.; Pettus, T. R. R. Tetrahedron 2002, 58, 5367-5405.

[6] (a) Xu, Z. X.; Huang, K.; Liu, T.; Xie, M. J.; Zhang, H. B. Chem. Commun. 2011, 4923-4925. (b) Liang, J. X.; Chen, J. B.; Liu, J. P.; Li, L.; Zhang, H. B. Chem. Commun. 2010, 3666-3668. (c)
Liang, J. X.; Chen, J. B.; Du, F. X.; Zeng, X. H.; Li, L.; Zhang, H. B. Org. Lett. 2009, 11, 2820-2823.

[7] Gilmore, C. D.; Allan, K. M.; Stoltz, B. M. J. Am. Chem. Soc. 2008, 130, 1558-1559.

[8] (a) Zhdankin, V. V.; Stang, P. J. Chem. Rev. 2008, 108, 52995358. (b) Richardson, R. D.; Wirth, T. Angew. Chem, Int. Ed. 2006, 45, 4402-4404. (c) Wirth, T. Angew. Chem. Int. Ed. 2005 , 44, 3656. (d) Moriarty, R. M. J. Org. Chem. 2005, 70, 2893-2903. (e) Zhdankin, V. V.; Stang, P. J. Chem. Rev. 2002, 102, 25232584.

[9] (a) Kita, Y.; Takada, T.; Gyoten, M.; Tohma, H.; Zenk, M. H.; Eichhorn, J. J. Org. Chem. 1996, 61, 5857-5864; (b) Kodama, S.; Hamashima, Y.; Nishida, K.; Node, M. Angew. Chem. Int. Ed. 2004, 43, 2659-2661.

[10] (a) Sha, C. K.; Young, J. J.; Yeh, C. P.; Chang, S. C.; Wang, S. L. J. Org. Chem. 1991, 56, 2694-2696. (b) Li, W. D. Z.; Wang, X. W. Org. Lett. 2007, 9, 1211-1214.

[11] (a) Tsuda, Y.; Nakai, A.; Ito, K.; Suzuki, F.; Haruna, M. Heterocycles 1984, 22, 1817-1820. (b) Joo, J. M.; David, R. A.; Yuan, Y.; Lee, C. Org. Lett. 2010, 12, 5704-5707. (c) Zhang, F.; Simpkins, N. S.; Wilson, C. Tetrahedron Lett. 2007, 48, 59425947. (d) Pearson, W. H.; Kropf, J. E.; Choy, A. L.; Lee, I. Y.; Kampf, J. W. J. Org. Chem. 2007, 72, 4135-4148. (e) Gao, S.; Tu, Y. Q.; Hu, X.; Wang, S.; Hua, R.; Jiang, Y.; Zhao, Y.; Fan, X.; Zhang, S. Org. Lett. 2006, 8, 2373-2376. (f) Wang, Q.; Padwa, A. Org. Lett. 2006, 8, 601-604. (g) Padwa, A.; Wang, Q. J. Org. Chem. 2006, 71, 7391-7402. (h) Kim, G.; KimandK, J. H.; Lee, Y. J. Org. Chem. 2006, 71, 2185-2187. (i) Stanislawski, P. C.; Willis, A. C.; Banwell, M. G. Org. Lett. 2006, 8, 2143 2146. (j) Allin, S. M.; Streetley, G. B.; Slater, M.; James, S. L.; Martin, W. P. Tetrahedron Lett. 2004, 45, 5493-5496. (k) Padwa, A.; Lee, H. I.; Rashatasakhon, P.; Rose, M. J. Org. Chem. 2004, 69, 8209-8218. (1) El Bialy, S. A. A.; Braun, H.; Tietze, L. F. Angew. Chem. Int. Ed. 2004, 43, 5391-5393. (m) Yasui, Y.; Suzuki, K.; Matsumoto, T. Synlett 2004, 619-622. 